\title{
Study of Defect Modes in Photonic Band Structure of Magnetic Photonic Crystals by Means of Green's Function
}

\author{
N. $\operatorname{ANSARI}^{a}$ AND M.M. TEHRANCHI ${ }^{a, b, *}$ \\ ${ }^{a}$ Laser and Plasma Research Institute, G.C. \\ Shahid Beheshti University, Evin, Tehran 1983963113, Iran \\ ${ }^{b}$ Physics Department, G.C., Shahid Beheshti University \\ Evin, Tehran 1983963113, Iran
}

\begin{abstract}
In this paper, we create an analytical model to investigate the localized defect modes associated with a defect cell inserted into a one-dimensional magnetic photonic crystal. The structure is a magnetic superlattice with alternative layers of two different magnetic permeability containing a defect cell which is a layer of different nature (material or/and size) from the other layers. This leads to appearance of several localized defect modes within the magnetic photonic band gap. Our analytical approach is based on the transfer matrix and Green's function methods to calculate the frequency and number of the defect modes which can be controlled easily by varying parameter values of the constituent layers of the magnetic photonic crystal. This method results in an analytical formula for prediction of frequency of the defect modes for both TE and TM polarizations at arbitrary angle of incidence.
\end{abstract}

PACS numbers: 75.70.Cn, 42.70.Qs, 84.30.Vn, 85.70.Sq

\section{Introduction}

Recently, interesting results of investigations of photonic periodic structures such as magnetic photonic crystals (MPCs) have received attractive attention. MPCs are artificial periodic structures comprising alternating layers with different magnetic properties or magnetic/non-magnetic materials [1]. In such structures, there is a range of frequency spectrum where light (photon) propagation is prohibited which is nominated as magnetic photonic band gaps (MPBGs). MPBG can be obtained when both the dielectric permittivity $(\varepsilon)$ and the magnetic permeability $(\mu)$ vary in the MPCs [2]. Noticeably, the employments of magnetic materials in periodic structures allow the tuning of MPBG due to the dependence of their optical properties on an external magnetic field and temperature $[3,4]$.

By breaking the periodicity of structure or by introducing a disorder into the regular structure of a MPC, allowed modes of electromagnetic waves can be created inside the MPBG, called defect modes. So it is possible to tune the defect modes to any frequency in the gaps by designing the size and material of the defect layer. The defect modes play important roles in the application of MPCs to high efficiency isolators, waveguides and filters, etc.

\footnotetext{
* corresponding author; e-mail: teranchi@cc.sbu.ac.ir
}

However, the design of controllable defect modes in MPCs requires predictive formulae for the frequency dependence of the defect modes on physical parameters of MPCs and on polarization and angle of incident light. In this paper, the equation governing the frequencies of the defect modes, perturbed by a defect cell, is derived analytically for an infinite 1D MPC consisting of the periodically repeated bilayer cells. For this purpose, we use the Bloch periodic condition and introduce a Green function to solve the equations obtained from transfer matrix method (TMM). The frequencies and the number of localized defect modes depend upon the layers materials and thicknesses. We have assumed that the dielectric and magnetic absorptions of the layers are negligible and $\varepsilon$ and $\mu$ are to be constant and ignore any anisotropy in $\varepsilon$ and $\mu$. Then we calculate the MPBG and defect mode frequencies for both transverse electric (TE) and transverse magnetic $(\mathrm{TM})$ polarizations at various angles of incidence.

\section{Theoretical formalism}

Let us consider an infinite 1D MPC, comprising the periodically repeated thin layers of two types, i.e. $\ldots \mathrm{ABABAB} \ldots$ The layered structure is periodic in the $z$ direction and homogeneous in the $x-y$ plane. The layers are characterized by their thicknesses, $d_{\mathrm{A}}$ and $d_{\mathrm{B}}$, dielectric permittivities $\varepsilon_{\mathrm{A}}$ and $\varepsilon_{\mathrm{B}}$, the magnetic permeabilities $\mu_{\mathrm{A}}$ and $\mu_{\mathrm{B}}$, respectively, and $\Lambda=d_{\mathrm{A}}+d_{\mathrm{B}}$ is the length of periodicity of the MPC. A defect is modeled as 
a double layer situated at the center of the MPC. The defect layers are characterized by their thickness $d_{X}$ and $d_{Y}$, dielectric permittivities $\varepsilon_{X}$ and $\varepsilon_{Y}$, and magnetic permeabilities $\mu_{X}$ and $\mu_{Y}$. Here we consider that $\varepsilon$ has equal value for all layers.

Let a plane wave be injected into the MPC at an incident angle of $\theta_{\mathrm{A}}$ with $+z$ direction. In this case, the electric field can be written as

$$
\boldsymbol{E}(\boldsymbol{r})=c_{J}^{+} \mathrm{e}^{\mathrm{i} \boldsymbol{k}_{J} \cdot \boldsymbol{r}}+c_{J}^{-} \mathrm{e}^{-\mathrm{i} \boldsymbol{k}_{J} \cdot \boldsymbol{r}},
$$

in which the magnitude of wave vector, $\boldsymbol{k}_{J}=k_{J} \sin \theta_{J} \hat{x}+$ $k_{J} \cos \theta_{J} \hat{z}$, for a wave with a frequency of $\omega$ propagating in the $J$-th layer (i.e. $J=\mathrm{A}, \mathrm{B}, X$ and $Y$ ) with a refractive index of $n_{J}=\sqrt{\varepsilon \mu_{J}}$, is $k_{J}=\frac{n_{J} \omega}{c}$ where $c$ is the speed of light in vacuum. In Eq. (1), $\boldsymbol{r}$ is the position vector and $c_{J}^{+}\left(c_{J}^{-}\right)$is the amplitude vector of the incident (reflected) electric field in the $J$-th layer. Using the Maxwell equations and Eq. (1) a similar equation for the magnetic field can be derived. For the TE (TM) wave, the electric field $E$ (the magnetic field $H$ ) is in the $y$ direction. At the interface of adjacent layers, $E$ and $H$ should be continuous. It has been derived that $E$ and $H$ between the $(I+1)$-th and $I$-th unit cells are related by the transfer matrix $T_{\mathrm{N}}$ for a normal cell $(I \neq 0)$ and $T_{\mathrm{D}}$ for the defective cell $(I=0)$. The transfer matrix for a normal cell is given by [5]:

$$
T_{\mathrm{N}}=\left(\begin{array}{cc}
\lambda_{\mathrm{N}} & \sigma_{\mathrm{N}} \\
\xi_{\mathrm{N}} & \eta_{\mathrm{N}}
\end{array}\right),
$$

in which

$$
\begin{aligned}
& \lambda_{\mathrm{N}}=\cos \alpha_{\mathrm{A}} \cos \alpha_{\mathrm{B}}-\frac{p_{\mathrm{B}}}{p_{\mathrm{A}}} \sin \alpha_{\mathrm{A}} \sin \alpha_{\mathrm{B}}, \\
& \sigma_{\mathrm{N}}=\frac{\mathrm{i}}{p_{\mathrm{B}}} \cos \alpha_{\mathrm{A}} \sin \alpha_{\mathrm{B}}+\frac{\mathrm{i}}{p_{\mathrm{A}}} \cos \alpha_{\mathrm{B}} \sin \alpha_{\mathrm{A}}, \\
& \xi_{\mathrm{N}}=\mathrm{i} p_{\mathrm{B}} \cos \alpha_{\mathrm{A}} \sin \alpha_{\mathrm{B}}+\mathrm{i} p_{\mathrm{A}} \cos \alpha_{\mathrm{B}} \sin \alpha_{\mathrm{A}}, \\
& \eta_{\mathrm{N}}=\cos \alpha_{\mathrm{A}} \cos \alpha_{\mathrm{B}}-\frac{p_{\mathrm{A}}}{p_{\mathrm{B}}} \sin \alpha_{\mathrm{A}} \sin \alpha_{\mathrm{B}},
\end{aligned}
$$

where $\alpha_{\mathrm{A}}=\frac{2 \pi}{\Lambda} W \sqrt{\varepsilon \mu_{\mathrm{A}}} d_{\mathrm{A}} \cos \theta_{\mathrm{A}}, \alpha_{\mathrm{B}}=\frac{2 \pi}{\Lambda} W \sqrt{\varepsilon \mu_{\mathrm{B}}} d_{\mathrm{B}} \times$ $\cos \theta_{\mathrm{B}}, W=\omega \Lambda / 2 \pi c$ which is normalized frequency and the angles $\theta_{\mathrm{A}}$ and $\theta_{\mathrm{B}}$, determined by Snell's law of refraction, are the propagation angles for layers $\mathrm{A}$ and $\mathrm{B}$. For the TE polarization $p_{\mathrm{A}}$ and $p_{\mathrm{B}}$ are given by $p_{\mathrm{A}}=$ $\frac{1}{c \mu_{0}} \sqrt{\frac{\varepsilon}{\mu_{\mathrm{A}}}} \cos \theta_{\mathrm{A}}$ and $p_{\mathrm{B}}=\frac{1}{c \mu_{0}} \sqrt{\frac{\varepsilon}{\mu_{\mathrm{B}}}} \cos \theta_{\mathrm{B}}$ and for the TM polarization, they are given by $p_{\mathrm{A}}=\frac{1}{c \mu_{0}} \sqrt{\frac{\varepsilon}{\mu_{\mathrm{A}}}} \frac{1}{\cos \theta_{\mathrm{A}}}$ and $p_{\mathrm{B}}=\frac{1}{c \mu_{0}} \sqrt{\frac{\varepsilon}{\mu_{\mathrm{B}}}} \frac{1}{\cos \theta_{\mathrm{B}}}$ where $\mu_{0}$ is the magnetic permeability of vacuum. The transfer matrix $T_{\mathrm{D}}$ related to the defect cells can also be written similarly as

$$
T_{\mathrm{D}}=\left(\begin{array}{cc}
\lambda_{\mathrm{D}} & \sigma_{\mathrm{D}} \\
\xi_{\mathrm{D}} & \eta_{\mathrm{D}}
\end{array}\right),
$$

where the matrix elements are defined as those introduced for Eq. (2) in terms of parameters relevant to the defect layers.

In a structure with an infinite number of layers, assumption of translational symmetry aided by the Bloch theorem together with the use of TMM, one can obtain a transcendental equation determining the band structure, namely [6]:

$$
2 \cos (\kappa \Lambda)=\lambda_{\mathrm{N}}+\eta_{\mathrm{N}} \equiv F_{\mathrm{N}}(\omega) .
$$

In this equation, $\kappa$ is the Bloch wave number. The problem of finding defect mode frequencies within the spectral band gaps is according to that considered by Tamura in Ref. [7], but in the case of a phononic crystal. In this order, by applying boundary conditions for electric and magnetic fields of the electromagnetic waves at the interface of MPC layers and implementing some mathematical procedures, a recursion relation for the electric field at the surface of each unit cell can be obtained. It is possible to write this relation as the sum of two terms; one of them is merely related to the perfect MPC and the other one is due to the existence of the defect. Subsequently, considering an infinite perfect MPC and applying the Green function method, an analytical relation for defect frequencies can be deduced. The defect mode frequencies are given by solutions of equation,

$$
g_{0} F_{\mathrm{D}}-g_{1}\left(\lambda_{\mathrm{N}} \eta_{\mathrm{D}}+\lambda_{\mathrm{D}} \eta_{\mathrm{N}}-\sigma_{\mathrm{N}} \xi_{\mathrm{D}}-\sigma_{\mathrm{D}} \xi_{\mathrm{N}}\right)=0,
$$

where $F_{\mathrm{D}}=\lambda_{\mathrm{D}}+\eta_{\mathrm{D}}$, and $g_{0}$ and $g_{1}$ are given by $g_{0}=\mp \frac{1}{\sqrt{F_{\mathrm{N}}^{2}-4}}$ and $g_{1}=\mp \frac{1}{\sqrt{F_{\mathrm{N}}^{2}-4}}\left(\frac{\mp \sqrt{F_{\mathrm{N}}^{2}-4}+F_{\mathrm{N}}}{2}\right)$ where the minus (plus) sign is applied for $F_{\mathrm{N}}>2\left(F_{\mathrm{N}}<-2\right)$. Thus, each defect mode frequency locates only in the MPBGs. Consequently, Eq. (6) determines the frequencies and the number of the localized modes in each MPBG for different parameter values of the MPC and the defect bilayer.

\section{Results and discussion}

Let us consider an MPC whose parameters have been selected according to Ref. [8]. The magnetic permeability and the thickness of the two layers are as follows: $\mu_{\mathrm{A}}=1$, $\mu_{\mathrm{B}}=4$, and $d_{\mathrm{A}}=0.8 \Lambda$, and $d_{\mathrm{B}}=0.2 \Lambda$. For all layers $\varepsilon$ is constant and has real value of 4 . The defect layer is considered with $\mu_{X}=4$ and $d_{X}=0.4 \Lambda$.

Figure 1 shows normalized frequency as a function of propagating angle $\theta_{\mathrm{A}}$. For normal incident, both TE and TM modes are equivalent. The white areas represent the propagation bands and the gray areas are the forbidden bands. At the first band structure, with increasing oblique angle the gap width of TM mode increases, whereas gap width of TE mode decreases up to the Brewster angle $\left(\theta_{\mathrm{Br}}\right)$ and then grows up again. The $\theta_{\mathrm{Br}}$ which is $\tan ^{-1}\left(\sqrt{\frac{\mu_{\mathrm{B}}}{\mu_{\mathrm{A}}}}\right)$ corresponds to the Brewster angle where there is no reflection of TE waves and therefore there is no band gap. Defect branches (defect modes) can be seen in Fig. 1 within the band gaps as dashed lines. At the gap closing points there is no defect mode.

Another interesting property is that the localized defect modes appear only in the band gap, which depends upon defect permeability, thickness and oblique angle of incident waves. The effect of variation of these parameters on the defect mode frequencies for normal 


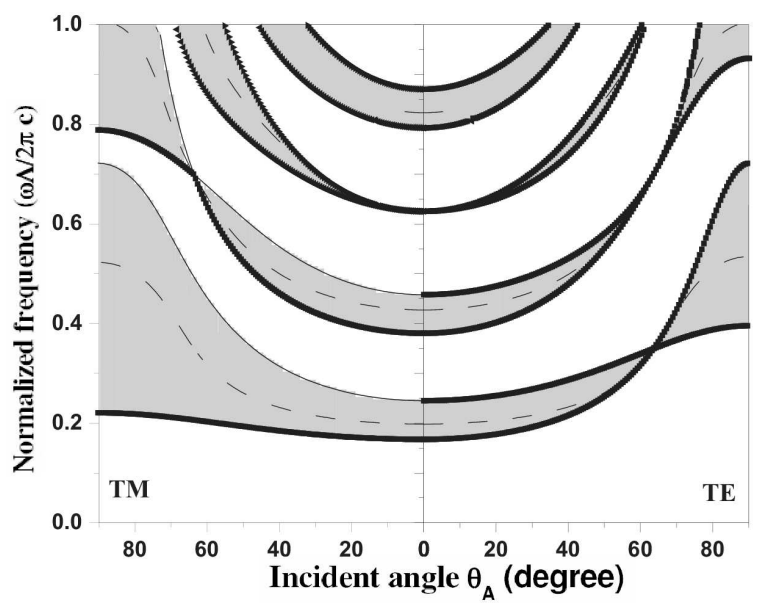

Fig. 1. MPBG in terms of normalized frequency for the incident angle $\theta_{\mathrm{A}}$.
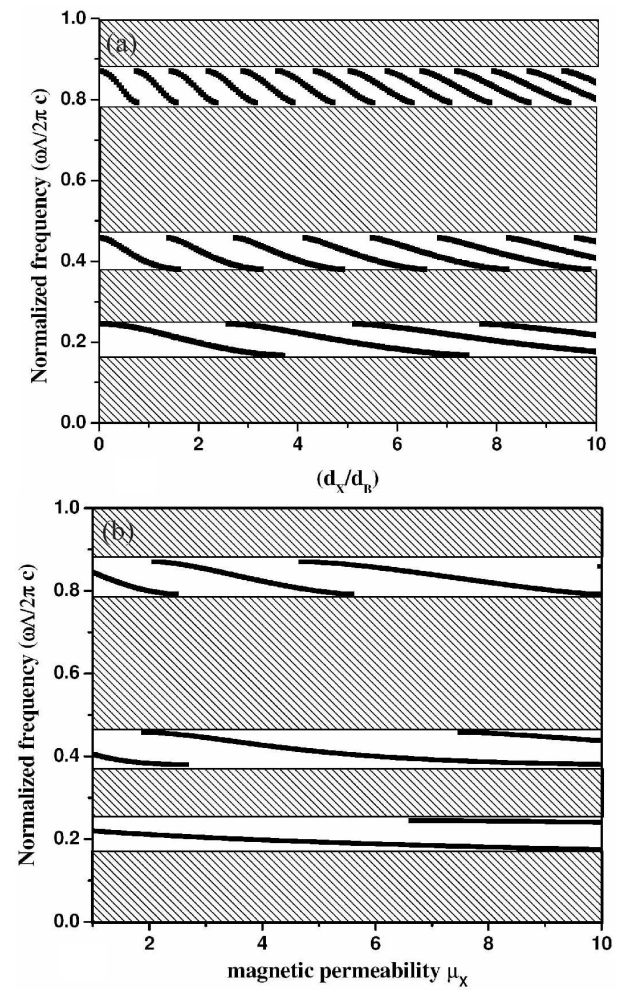

Fig. 2. The defect mode frequency versus defect thickness (a) and magnetic permeability (b). incident is represented in Fig. 2. The white areas represent the forbidden bands and the cross-hatched areas are the propagation bands. By increasing the defect thickness or magnetic permeability, the frequencies of the localized modes move from the higher propagation band to the lower one. Moreover, manipulating these parameters provides a range of values suitable for designing multi-frequency filters. As indicated, the number of branches of the defect modes in the second band gap is more than their number in the first one.

\section{Conclusion}

The present paper demonstrated an analytical study of the localized defect modes in the MPBG of a 1D MPC for both TE and TM polarization at arbitrary angle of incidence. By using the Green function method to solve the equations obtained by TMM, the dispersion curve of magnetic periodic structures has been calculated in detail. By increasing the defect thickness or magnetic permeability, the frequencies of the localized modes move from the higher propagation band to the lower one. Then, the dependence of the frequencies of the localized modes on the defect thickness and magnetic permeability emerging in the MPBG was looked into in order to show its multifunctional applications in MPCs.

\section{References}

[1] I.L. Lyubchanskii, N.N. Dadoenkova, M.I. Lyubchanskii, E.A. Shapovalov, Th. Rasing, J. Phys. D, Appl. Phys. 36, R277 (2003).

[2] K.S. Srivastava, S.P. Ojha, Optik - International Journal for Light and Electron Optics, in press.

[3] M.M. Sigalas, C.M. Soukoulis, R. Biswas, K.M. Ho, Phys. Rev. B 56, 959 (1997).

[4] C.S. Kee, J.E. Kim, H.Y. Park, I. Park, H. Lim, Phys. Rev. B. 61, 15523 (2000).

[5] M. Born, E. Wolf, Principles of Optics, 7th ed., Cambridge University Press, New York 1999, p. 61.

[6] P. Yeh, Optical Waves in Layered Media, Wiley, New York 2005, p. 124.

[7] S. Tamura, Phys. Rev. B 39, 1261 (1989).

[8] Z. Wang, D. Liu, Appl. Phys. B 86, 473 (2007). 